\title{
MODULAR INVARIANTS OF A GENERAL SYSTEM OF LINEAR FORMS
}

\author{
By L. E. Dickson.
}

[Received February 2nd, 1909.-Read February 11th, 1909.]

1. After giving an outline of a method of marked simplicity for the investigation of modular invariants, we state the main results of the present self-contained treatment of linear forms.

In the simplest case, the coefficients of the forms discussed are integers reduced modulo $p$, where $p$ is a prime. In the general case, the coefficients are Galois' imaginaries $c_{0}+c_{1} \rho+\ldots+c_{n-1} \rho^{n-1}$, in which the $c$ 's are integers reduced modulo $p$, while $\rho$ is a root of a congruence of degree $n$, irreducible modulo $p$. These $p^{n}$ imaginaries constitute the Galois field $G F\left[p^{n}\right]$.

We consider the system $L$ of $q$ linear forms,

$$
l_{i}=a_{i 1} x_{1}+\ldots+a_{i m} x_{n} \quad(i=1, \ldots, q),
$$

whose coefficients are arbitrary elements of the $G F\left[p^{n}\right]$. Let $G$ be any given group of linear homogeneous transformations on $x_{1}, \ldots, x_{m}$ with coefficients in the field. The $p^{4 m n}$ particular systems $L^{\prime}, L^{\prime \prime}, \ldots$, obtained by assigning to the $a$ 's particular values, can be separated into certain classes $C_{0}, C_{1}, \ldots, C_{f-1}$ under the group $G$, such that two systems are transformable into each other by transformations of $G$ if, and only if, the systems belong to the same class.

By a general theorem on interpolation, there exists one, and but one, polynomial $I(a)$ in $a_{11}, a_{12}, \ldots, a_{q m}$, with each exponent $\leqslant p^{n}-1$ and coefficients in the $G F\left[p^{n}\right]$, such that $I(a)$ takes a prescribed value $v_{a}$ for each set of elements $a$ in the field. In particular, if $v_{a}$ is the same for all sets $a$ leading to a class $C$, so that $I(a)$ takes prescribed values $v_{0}, v_{1}, \ldots, v_{f-1}$ for the respective classes $C_{0}, C_{1}, \ldots, C_{f-1}$, then $I(a)$ is obviously an invariant of the system of forms (1) under the group $G$. Thus $I(a)=\Sigma v_{k} I_{l}$, where $I_{k}$ is the uniquely determined invariant which has the value unity for the class $C_{k}$ and the value zero for every class $C_{i}(i \neq k) ; I_{k}$ is called the characteristic invariant for the class $C_{k}$. If $\Sigma d_{k} I_{k}=0$, each $d_{k}=0$. Although there is no linear homogeneous relation between the $I$ 's, we have $\Sigma I_{k}=1$. In the former sense, we 
shall say that $I_{0}, \ldots, I_{f-1}$ are linearly independent. Hence the total number of linearly independent invariants under $G$ equals the number of classes under $G$.

When $G$ is the total linear group on the $m$ variables, the invariants just discussed are the absolute invariants of the system $L$. When the group is the group $G_{1}$ of all transformations of determinant unity, those invariants of $G_{1}$ which are multiplied by $\Delta^{w}$ under every transformation of determinant $\Delta$ are the relative invariants of weight $w$ (absolute when $w$ is a multiple of $p^{n}-1$ ). The number $N$ of linearly independent invariants, relative and absolute, of the system $L$ obviously does not exceed the number of the linearly independent invariants of $G_{1}$, the latter being the number $f$ of the classes under $G_{1}$. As a matter of fact, $N=f$, as I have shown by a rather technical proof.* This result, however, is not presupposed in the present paper. Indeed, we here exhibit explicitly $f$ linearly independent invariants of the general system $L$ of linear forms. Hence $N \geqslant f$. But $N \leqslant f$, by the above simple discussion; whence $N=f$.

In the algebraic theory, $q$ linear forms in $m$ variables have no rational integral invariants if $q<m$, while, if $q \geqslant m$, the invariants are functions of the determinants of sets of $m$ forms. If, for $h<m, l_{1}, \ldots, l_{h}$ are linearly independent, and

$$
l_{h+1}=c_{1} l_{1}+\ldots+c_{h} l_{h},
$$

the $c$ 's are obviously invariant under linear transformation; in the modular theory (in contrast to the algebraic theory), the $c$ 's may be expressed by rational integral invariants $(\$ 8)$. In spite of the greater variety of invariants in the modular theory, we establish the following fundamental theorem: Every invariant of a system of $q>m$ linear forms in $m$ variables is a rational integral function of the invariants of systems of $m$ forms in $m$ variables.

2. When there is a single variable, $G_{1}$ contains only the identity transformation, so that each system of forms $a_{i 1} x_{1}$ constitutes a class. As a complete system of linearly independent invariants we may take the $p^{4 n}$ products of the powers of the $a_{i 1}$ with exponents $0,1, \ldots, p^{n}-1$. Henceforth we take $m>1$.

For a single form $l_{i}$ there are two classes under $G_{1}$, one class containing only $l_{i} \equiv 0$, the other class containing the forms in which not every

* "General Theory of Modular Invariants," 'Transactions of the American Mathematical Society, Vol. x., April, 1909. 
$a_{i r}$ is zero and hence conjugate with $:_{1}$. For the respective classes the characteristic invariants are $A_{i}$ and $1-1_{i}$, where

$$
d_{i}=\prod_{r-1}^{\prime \prime \prime}\left(1-a_{i n}^{\mu}\right) \quad\left(\mu=p^{n}-1\right) .
$$

3. Two forms $l_{i}, l_{j}$ may be lineirly independent, or dependent with $l_{i} \neq 0$, sc. Hence they can be transformed within $G_{1}$ into one of the four pairs in the following table, which also gives the values of certain invariants :-

\begin{tabular}{|c|c|c|c|c|c|}
\hline$l_{i}$ & $l_{j}$ & $A^{i}$ & $A_{j}$ & $A_{i} A_{i}$ & $V_{i j}$ \\
\hline$x_{1}$ & $d \cdot x_{2}$ & 0 & 0 & 0 & 0 \\
$x_{1}$ & $c x_{1}$ & 0 & $1-c^{\mu}$ & 0 & $c$ \\
0 & $x_{1}$ & 1 & 0 & 0 & 0 \\
0 & 0 & 1 & 1 & 1 & 0 \\
\hline
\end{tabular}

Here $d=1$, if $m>2$; while, for $m=2, d$ is the non-vanishing determinant $D_{i j}$ of a pair of independent forms $l_{i}, l_{j}$. For the construction of an invariant $V_{i j}$ taking the assigned values for the various classes, we may proceed as in $\$ 8$ or as follows. If, in (2), we replace $a_{i r}$ by $a_{i v}-\kappa a_{j i}$ and apply

$$
\kappa^{r^{n}}=\kappa,
$$

we obtain a polynomial in $\kappa$ of degree $\mu=p^{n}-1$; the coefficient of each power of $\kappa$ is au absolute invariant of $l_{i}, l_{j}$. It will be seen to be convenient to separate the terms involving $\kappa^{\mu}$ and write

$$
I_{i=1}^{\prime \prime}\left[1-\left(a_{i v}-\kappa a_{j r}\right)^{\mu}\right] \equiv A_{i}+A_{i}\left(A_{j}-1\right) \kappa^{\mu}-\sum_{t=1}^{\mu} S_{i j t} \kappa^{t}
$$

for every root of (3). For $x_{1}, c x_{1}$, the non-vanishing $a$ 's are $a_{i 1}=1$, $a_{j 1}=c$. Hence in (t) the factors with $v>1$ equal unity, while the factor with $v=1$ is $1-(1-\rho c)^{\mu}$. But

$$
(\alpha-\beta)^{\mu} \equiv \sum_{t=0}^{\mu} \alpha^{\mu-t} \beta^{t} \quad(\bmod p) .
$$

Hence, for the pair $x_{1}, c x_{1}$, (4) becomes

$$
-\sum_{i=1}^{\mu} c^{t} \kappa^{t}=-\sum_{t-1}^{\mu} S_{i, j} \kappa^{t}
$$


For the remaining pairs in the table, we find immediately that $S_{i j t}=0$. Hence $V_{i j}=S_{i j 1}, \quad S_{i j t}=S_{i j 1}$. Thus $V_{i j}$ is the sum of the coefficients of $\kappa, \kappa^{\mu+1}, \kappa^{2 \mu+1}, \ldots$ in the expansion of the left member of (4). Applying (5), get

$$
\begin{gathered}
V_{i j}=\Sigma F_{1 t_{1}} F_{2 t_{2}} \ldots F_{m t_{m}} \\
{\left[t_{1}+\ldots+t_{m} \equiv 1(\bmod \mu) ; t_{1}, \ldots, t_{m}=0,1, \ldots, \mu\right],} \\
F_{v t}=-a_{i v}^{\mu-t} a_{j v}^{t}(t>0), \quad F_{v 0}=1-a_{i v}^{\mu} .
\end{gathered}
$$

In particular, for $m=2$,

$$
V_{i j}=\left(a_{i 1}^{\mu}-1\right) a_{i 2}^{\mu-1} a_{j 2}+\left(a_{i 2}^{\mu}-1\right) a_{i 1}^{\mu-1} a_{j 1}+\sum_{i=1}^{\mu} a_{i 1}^{\mu-t} a_{j 1}^{t} a_{i 2}^{t-1} a_{j 2}^{\mu+1-t} .
$$

By undetermined coefficients it follows, from the above table (or by the more instructive method of $\S 4$ ), that the absolute invariants

$$
1, A_{i}, A_{j}, A_{i} A_{j}, V_{i j}^{t} \quad(t=1, \ldots, \mu)
$$

are linearly independent; also, that, for $m=2$, the only linear relation between the invariants $(8)$ and $D_{i j}^{k}(k=1, \ldots, \mu)$ is

$$
D_{i j}^{\mu}+V_{i j}^{\mu}=\left(1-A_{i}\right)\left(1-A_{j}\right) \text {. }
$$

The number of classes is $p^{n}+3$ if $m>2,2 p^{n}+1$ if $n=2$.

Theorex.-As a complete set of linearly independent invariants of two linear forms $l_{i}$ and $l_{j}$ in the $G F\left[p^{n}\right]$ on $m$ variables, we may take the $p^{n}+3$ absolute invariants (8) if $m>2$, and, if $m=2$, the $2 p^{n}+1$ invariants

$$
A_{i}, A_{j}, A_{i} A_{j}, V_{i j}^{t}, D_{i j}^{t} \quad\left(t=1, \ldots, p^{n}-1\right)
$$

4. We readily derive the characteristic invariants for the various classes of two linear forms in $m$ variables. For $x_{1}, 0 ; 0, x_{1} ; 0,0$, these are

$$
A_{j}-A_{i} A_{j}, \quad A_{i}-A_{i} A_{j}, \quad A_{i} A_{j} .
$$

For $x_{1}, c x_{1}$, where $c \neq 0$, the characteristic invariant is

$$
-\sum_{k=1}^{\mu} c^{-k} V_{i j}
$$

Indeed, for $V=c$, this sum is $-\mu \equiv 1(\bmod p)$, for $V=\gamma \neq c$; it equals a fraction with the numerator $\left(c^{-1} \gamma\right)^{\mu+1}-c^{-1} \gamma$, which vanishes in the field. For $m=2$, the characteristic invariant of $x_{1}, d x_{2}(d \neq 0)$ is likewise

$$
-\sum_{k=1}^{\mu} d^{-k} D_{i j}^{k}
$$

SER. 2. VOL. 7. No. 1032 . 
The linear independence of invariants (10) thus follows from that of the characteristic invariants (11), (12), (13), where $c$ and $d$ range over the $\mu$ elements $\neq 0$ of the field. The sum of the $\mu$ functions (12) is $V_{i j}^{\mu}$, since* the sum of the $k$-th powers of the elements of the field is zero for $0<k<\mu$, and -1 for $k=\mu$. But the sum of all the characteristic invariants is $1(\S 1)$. Hence relation (9) follows.

For $m>2$, the characteristic invariant of $x_{1}, x_{2}$ is

$$
\left(1-A_{i}\right)\left(1-A_{j}\right)-V_{i j}^{\mu}
$$

determined so that the sum of all shall be unity, or directly from the table of $\$ 3$. Since the linearly independent characteristic invariants (11), (12), (14) are linear functions of invariants (8), the latter are linearly independent.

5. For a given set of elements $E$, we shall say that

$$
E_{r s t \ldots .} \text { precedes } E_{\rho \sigma \tau \ldots}, \quad E_{\rho \sigma \ldots} \text { follows } E_{r s . \ldots},
$$

if $r<\rho$; or, if $r=\rho, s<\sigma$; or, if $r=\rho, s=\sigma, t<\tau$; \&c.

6. Every invariant of $q$ binary linear forms will be shown to equal a rational integral function of $A_{i}, V_{i j}, D_{i j}(i, j=1, \ldots, q)$. We employ a canonical type for each system of $q$ forms with given coefficients in the field.

First, let not every $D_{i j}$ vanish. Let $D_{r s} \neq 0(r<s)$, but let every preceding $D_{i j}(i<j)$ vanish, viz.,

$$
D_{i j}=0(i<r), \quad D_{r i}=0(r<k<s), \quad D_{r s} \neq 0 .
$$

After applying an obvious transformation of $G_{1}$, we have

$$
l_{r}=x_{1}, \quad l_{s}=c x_{2}, \quad c=D_{r s} .
$$

By $D_{i r}=D_{i s}=0(i<v), l_{i}$ is free of $x_{2}$ and $x_{1}$. By $D_{r k}=0, l_{k}$ is free of $x_{2}$. Hence the canonical type is

$$
\begin{array}{ll}
l_{i}=0, \quad l_{r}=x_{1}, \quad l_{k}=c_{k} x_{1}, \quad l_{3}=c x_{2}, \quad l_{t}=-d_{t} x_{1}+e_{t} \cdot c_{2} \\
& (i<r<k<s<t<q ; c \neq 0) .
\end{array}
$$

Next, let every $D_{i j}=0$, but not every $l_{i}$, vanish identically. Let $l_{f}$ be the first non-vanishing $l$. Applying a transformation of $G_{1}$, we get

$$
l_{i}=0, \quad l_{f}=x_{1}, \quad l_{j}=m_{j} x_{1} \quad(i<f<j \leqslant q) .
$$

- Dickson, Linear Groups, Leipzig, 1901, p. 54. 
Finally, there remains the system

$$
l_{i}=0 \quad(i=1, \ldots, q) .
$$

A system of forms (17) defines a class $A_{r s}^{\text {cle }}$; a system (18) defines a class $B_{f}^{m}$; the system (19) the class $C_{0}$. In addition to the definition in $\S 5$, we shall say that any class $A$ precedes a class $B$ or $C_{0}$, and that any $B$ precedes $C_{0}$. No two classes are equivalent under $G_{1}$. As to the $A$ 's, this follows from the invariance of the $D_{i j}$; for (17), we have relations (16) and

$$
D_{r s}=c, \quad D_{r t}=e_{l}, \quad D_{k s}=c c_{l}, \quad D_{s t}=c d_{l} \quad(r<k<s<t),
$$

so that the $c, d, e$ may be expressed in terms of invariants. For (18),

$$
V_{f j}=m j \quad(f<j \leqslant q),
$$

where $V$ is the invariant of $\$ 3$. Since the $c_{k}, d_{t}, e_{t}$ may take any values in the field and $c$ any values $\neq 0$, it follows from (20) that the products

$$
\prod_{k=r+1, \ldots, s-1 ; t=s+1, \ldots, q} D_{r s}^{\gamma} D_{l i s}^{\gamma_{k}} D_{r t}^{\epsilon_{t}} D_{s t}^{\delta_{t}} \quad\left(\gamma=1, \ldots, \mu ; \gamma_{k}, \epsilon_{t}, \delta_{t}=0,1, \ldots, \mu\right)
$$

are linearly independent in the field, and that the number of these invariants (22) is the number of classes $A_{r s}^{\text {cde }}$. In view of the factor $D_{r s}$, (22) vanishes for the classes $B, C_{0}$, and those classes $A_{\rho \sigma}$ which follow the $A_{r s}$. Similarly,

$$
1-A_{f}, \quad \prod_{j=f+1}^{l} V_{f j}^{\mu_{j}}\left(\dot{\mu}_{j}=0,1, \ldots, \mu ; \text { not every } \mu_{j}=0\right)
$$

are linearly independent, of the same number as the classes $B_{f}^{m}$, and vanish for the classes $C_{0}, B_{\phi}(\phi>f)$ which follow the $B_{f}$. Finally, with the class $C_{0}$ we associate the invariant 1 . We deduce at once the linear independence of the specified invariants whose number equals that of the classes.

Theовем-Every invariant of $q$ binary linear forms is expressible in terms of invariants of pairs of forms. A complete set of the

$$
\left(p^{n l}-1\right)\left(p^{n(1 / 1)}-1\right) /\left(p^{2 n}-1\right)+\left(p^{\prime \prime \prime}-1\right) /\left(p^{n}-1\right)+1
$$

linearly independent invariants of $q$ binary linear forms in the GF $\left[p^{n}\right]$ is given by unity, ${ }^{*}(22)$ and (23) for $r, s, f=1, \ldots, q ; r<s$.

7. For $q=3$, the $p^{3 n}+p^{2 n}+p^{n}+1$ linearly independent variants are

$$
\begin{array}{rll}
D_{12}^{\alpha} D_{13}^{\beta} D_{23}^{\gamma}(\alpha, \beta, \gamma=0,1, \ldots, \mu), & A_{1}, A_{2}, A_{3}, \\
V_{12}^{\rho}, V_{13}^{\rho}, V_{23}^{\rho}, V_{12}^{\rho} V_{13}^{\sigma} & (\rho, \sigma=1, \ldots, \mu) .
\end{array}
$$

* We may introduce $A_{1} A_{2}$ instead of 1 by (9). Then, if $q=2$, the set becomes (10). 
Other products can be expressed in terms of these by (9) and

$$
\begin{aligned}
& A_{1} D_{12}=0, \quad A_{1} D_{29}=\left(D_{12}^{\mu}-1\right)\left(D_{13}^{\mu}-1\right) D_{23}, \\
& A_{1} V_{12}=0, \quad A_{1} V_{23}^{t}=\left(D_{23}^{\mu}-1\right) D_{12}^{2 \mu-t} D_{13}^{t}-V_{12}^{2 \mu-t} V_{13}^{t}+V_{23}^{t}, \\
& D_{12} V_{12}=0, \quad D_{12}^{\lambda} V_{13}^{t}=(-1)^{\lambda} D_{12}^{\mu+\lambda-t} D_{23}^{t}\left(1-D_{13}^{\mu}\right) \text {, } \\
& V_{i j}^{t} V_{j k}^{e}=V_{i j}^{\mu+l-e} V_{i k}^{e}, \quad V_{i k}^{t} V_{j k}^{t}=V_{i j}^{2 \mu-e} V_{i k}^{t+e}, \\
& \left(1-A_{1}\right)\left(1-A_{2}\right)\left(1-A_{9}\right)=V_{12}^{\mu} V_{13}^{\mu}+D_{12}^{\mu} D_{13}^{\mu}+D_{12}^{\mu} D_{23}^{\mu}+D_{13}^{\mu} D_{23}^{\mu}-2 D_{12}^{\mu} D_{13}^{\mu} D_{23}^{\mu} \text {. } \\
& D_{12}^{\mu}, \quad 1-D_{12}^{\mu}, \quad\left(1-D_{12}^{\mu}\right)\left(1-D_{13}^{\mu}\right) .
\end{aligned}
$$

8. We employ a general function-theoretic process to construct an invariant, $V=V_{i_{1}, \ldots, i_{h+1}}$ of $h+1$ (not necessarily linear forms $l_{i_{1}}, \ldots, l_{i_{h+1}}$ ) in $m^{\prime}$ variables $\left(m^{\prime}>h\right)$, such that $V$ shall have the value $c_{1}$ when $l_{i_{1}}, \ldots, l_{i_{h}}$ are linearly independent, but $l_{i_{1}}, \ldots, l_{i_{h+1}}$ dependent and

$$
l_{i_{h+1}}=c_{1} l_{i_{1}}+\ldots+c_{h} l_{i_{h}} \text {, }
$$

while $V$ shall have the value zero fur all systems of $h+1$ forms not having the preceding two properties. Let $a_{11}, \ldots, a_{1 n}$ be the coefficients of $l_{1}$ in any order $; a_{i 1}, \ldots, a_{i m}$ the corresponding coefficients of $l_{i}$. Since $V$ shall vanish if the $h+1$ forms are linearly independent, we may set

$$
V=v \Pi\left(1-M \mu^{\mu}\right) \quad\left(\mu=p^{n}-1\right),
$$

$M$ ranging over the determinants of order $h+1$ in the matrix of the $l$ 's. It suffices to consider henceforth only sets of coefficients for which every $M=0$; for such a set $V=v$. Of these sets, consider one for which

$$
D=\left|a_{i j}\right| \quad\left(i=i_{1}, \ldots, i_{h} ; j=j_{1}, \ldots, j_{h}\right)
$$

is not zero, $j_{1}, \ldots, j_{h}$ being distinct integers $\leqslant m$. Thus there holds a relation of type (24), so that

$$
a_{i_{h_{+1}}}=\sum_{s=1}^{h} c_{s} a_{i_{s},} \quad(v=1, \ldots, m) .
$$

Taking $v=j_{1}, \ldots, j_{h}$, we have $h$ equations in which the determinant of the coefficients of $c_{1}, \ldots, c_{h}$ equals (26) with rows and columns interchanged. Let $d$ denote the determinant derived from (26) by replacing (in the first row) each $a_{i_{1} j}$ by $a_{i_{h+1} j}$. Then $I c_{1}=d$. Thus $c_{1}=d D^{\mu-1}$.

Let $D_{1}, \ldots, D_{r}$ denote the determinants (26), taken in any sequence, which are defined by the $r=\left(\begin{array}{c}m \\ h\end{array}\right)$ combinations $j_{1}, \ldots, j_{h}$ of $h$ integers

\footnotetext{
* The order of the intermediate subscripts $i_{2}, \ldots, i_{h}$ is immaterial.
} 
$\leqslant m$. Let $d_{1}, \ldots, d_{r}$ denote the corresponding determinants $d$ in the same sequence. We have

$$
v=d_{k} D_{k}^{\mu-1} \quad\left(\text { when } D_{k} \neq 0\right) .
$$

From $v=d_{1} D_{1}^{\mu-1}$, when $D_{1} \neq 0$, we get

$$
v \equiv d_{1} D_{1}^{\mu-1}+\lambda_{1}\left(1-D_{1}^{\mu}\right) \text {. }
$$

To determine $\lambda_{1}$, we consider a set making $D_{1}=0, D_{2} \neq 0$. For such a set

$$
d_{2} D_{2}^{\mu-1}=v=\lambda_{1} \text {, }
$$

so that for every set with $D_{1}=0$

$$
\lambda_{1}=d_{2} D_{2}^{\mu-1}+\lambda_{2}\left(1-D_{2}^{\mu}\right) .
$$

The product of the two members by $1-D_{1}^{\mu}$ are equal for every $D_{1}$. Thus

$$
v \equiv d_{1} D_{1}^{\mu-1}+\left(1-D_{1}^{\mu}\right) d_{2} D_{2}^{\mu-1}+\lambda_{2}\left(1-D_{1}^{\mu}\right)\left(1-D_{2}^{\mu}\right)
$$

for all sets. Proceeding similarly, we find by induction that

$$
\begin{array}{r}
v=d_{1} D_{1}^{\mu-1}+\left(1-D_{1}^{\mu}\right) d_{2} D_{2}^{\mu-1}+\left(1-D_{1}^{\mu}\right)\left(1-D_{2}^{\mu}\right) d_{3} D_{3}^{\mu-1}+\ldots \\
+\left(1-D_{1}^{\mu}\right) \ldots\left(1-D_{r-1}^{\mu}\right) d_{r} D_{r}^{\mu-1}
\end{array}
$$

with initially the additional term

$$
\lambda_{r}\left(1-D_{1}^{\mu}\right) \ldots\left(1-D_{r}^{\mu}\right) .
$$

But for a set for which $D_{1}, \ldots, D_{r}$ all vanish, (27) becomes $v=\lambda_{r}$, while by hypothesis $V=0$, so that, by (25), $v=0$. The invariance of $V$, given by (25) and (27), follows from the fact that it takes the same value for any two systems of $h+1$ forms equivalent under the total group.

For $h=1$ we set $i_{1}=i, i_{2}=j$, and obtain

$$
\begin{aligned}
V_{i j}=\left\{a_{j 1} a_{i 1}^{\mu-1}+\left(1-a_{i 1}^{\mu}\right) a_{j 2} a_{i 2}^{\mu-1}\right. & +\ldots \\
& \left.+\left(1-a_{i 1}^{\mu}\right) \ldots\left(1-a_{i m-1}^{\mu}\right) a_{j m} a_{i m}^{\mu-1}\right\} \Pi\left(1-M^{\mu}\right),
\end{aligned}
$$

where $M$ ranges over the determinants $a_{i r} a_{j s}-a_{i s} a_{j r}(r, s=1, \ldots, m)$.

9. We proceed to exhibit a complete set of linearly independent invariants of the system of $q \geqslant m$ linear forms $l_{i}$ on $m$ variables with arbitrary coefficients in the $G F\left[p^{n}\right]$. We shall employ a canonical type for each system with given coefficients.

First, let $m$ of the forms be linenrly independent. Let $l_{r_{1}}, \ldots, l_{r_{m}}$ $\left(r_{1}<r_{2} \ldots<r_{m}\right)$ be independent, but every preceding ( $\left.\S 5\right)$ set of $m$ forms dependent. Applying a transformation of $f_{1}$, we have

$$
l_{r}=x_{k} \quad(k<m), \quad l_{r_{m}}=c x_{m} \quad(c \neq 0) .
$$


For $i_{1}<r_{1}, l_{i_{1}}$ and any $m-1$ of the forms $l_{r_{1}}, \ldots, l_{r_{m}}$ are dependent by hypothesis; whence $l_{i_{1}}=0$. For $r_{1}<i_{2}<r_{2}, l_{r_{1}}, l_{i_{2}}$ and any $m-2$ of the forms $l_{r_{2}}, \ldots, l_{r_{m}}$ are dependent; whence $l_{i_{2}}$ is a multiple of $x_{1}$. Proceeding similarly, we get

$$
\begin{gathered}
l_{i_{1}}=0, \quad l_{i_{t}}=\sum_{j=1}^{t-1} b_{i_{t}} x_{j} \quad(t=2, \ldots, m+1), \\
\left(i_{1}<r_{1}<i_{2}<r_{2}<i_{3} \ldots<r_{m-1}<i_{m}<r_{m}<i_{m+1} \leqslant q\right) .
\end{gathered}
$$

Conversely, the $q$ forms $\left(28_{a}\right)$ and $\left(28_{b}\right)$ obviously have the properties that $l_{r_{1}}, \ldots, l_{r_{m}}$ are independent, while every preceding set of $m$ forms give dependent forms. We employ the determinants of order $m$ :

$$
\begin{aligned}
D_{r_{1} \ldots r_{m}}=c, \quad D_{r_{1} \ldots r_{j-1} i_{t} r_{j+1} \ldots r_{m}}=\left\{\begin{array}{cc}
c b_{i_{t} j} & (j<m) \\
b_{i_{i} j} & (j=m)
\end{array}\right\} \\
(t=2, \ldots, m+1 ; j<t) .
\end{aligned}
$$

The parameters $c, b$ are uniquely determined by these determinants, which are invariant. Hence no two of the classes* $A_{r_{1} \ldots r_{m}}^{b c}$ defined by (28) are equivalent under the group $G_{1}$. For given values of the $r$ 's, the number of these classes equals the number of products

$$
\Pi D_{r_{1} \ldots r_{m}}^{\gamma} D_{r_{1} \ldots r_{j-1} i_{t} r_{j+1} \ldots r_{m}}^{\beta_{i_{i} j}},
$$

where a particular product is obtained by taking all sets

$$
t=2, \ldots, m+1 ; j=1, \ldots, t-1,
$$

and then allowing $i_{2}, \ldots, i_{m+1}$ to range over all sets of integers $1, \ldots, q$ satisfying the inequalities $\left(28_{b}\right)$; while the various products are obtained by taking

$$
\gamma=1, \ldots, \mu ; \beta_{i_{t} j}=0,1, \ldots, \mu \quad\left(\mu=p^{n}-1\right) .
$$

Since the $b^{\prime}$ s are arbitrary in the field, while $c$ has any value $\neq 0$, it follows from (29) that the various products (30) are linearly independent in the field. Allowing the $r$ 's to vary, we obtain as many linearly in-

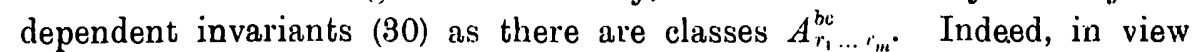
of the first factor, the product (30) vanishes for any class $A_{r_{1}^{\prime} \ldots r_{m}^{\prime}}^{b c^{\prime}}$ which follows $A_{r_{1} \ldots r_{m}}^{l c}$.

Next, for $h<m$, let $h$ of the $q$ forms be linearly independent, but every set of $h+1$ of the forms be dependent. Let

$$
l_{r_{i}}, \ldots, l_{r_{l i}} \quad\left(r_{1}<r_{2} \ldots<r_{h}\right)
$$

be independent, but every preceding set of $h$ forms dependent. Apply-

* We employ $b$ to denote the ordered aggregate of the coeffients in $\left(28_{b}\right)$. 
ing a transformation of $G_{1}$, we have $l_{r_{1}}=x_{1}, \ldots, l_{r_{h}}=x_{h}$. We derive $\left(28_{b}\right)$, with $m$ replaced by $h$. For the invariants determined in $\S 8$, we have

$$
V_{r_{j} r_{1} \ldots r_{j-1} r_{j+1} \ldots r_{h} i_{t}}=b_{i_{t} j} \quad(t=2, \ldots, h+1 ; j<t) .
$$

Hence no two of the present classes $B_{r_{1} \ldots r_{h}}^{b}$ are equivalent under $G_{1}$. Each invariant (31) vanishes for every subsequent class $B_{r_{1}^{\prime} \ldots r_{h^{\prime}}}^{l^{\prime}}\left(h^{\prime} \leqslant h\right)$ and for the class $C_{0}$ composed of the system (19). For given $r$ 's, consider the products

$$
\Pi V_{r_{j} r_{1} \ldots r_{j-1} r_{j+1} \ldots r_{h} i_{t}}^{\beta_{i_{i} j}} \text { (exponents not all zero), }
$$

a particular product being obtained by taking all sets $t=2, \ldots, h+1$; $j=1, \ldots, t-1$ and allowing $i_{2}, \ldots, i_{h+1}$ to range over all sets of $h$ of the integers $1, \ldots, q$ for which

$$
r_{1}<i_{2}<r_{2}<i_{9}<\ldots<r_{h}<i_{h+1} ;
$$

while the various products are obtained by taking each $\beta=0,1, \ldots, \mu$, but not all zero. In place of unity, excluded in (32), we desire an invariant $E$ which has the value 1 for each class $B_{r_{1} \ldots r_{h}}^{b}$ and the value 0 for the subsequent classes. Thus $E$ may be taken to be that invariant of the forms $l_{r_{1}}, \ldots, l_{r_{h}}$ alone which is characteristic for the class containing $x_{1}, \ldots, x_{h}$. Hence

$$
E_{r_{1} \ldots r_{h}}=1-\Pi\left(1-D^{\mu}\right),
$$

where $D$ ranges over the determinants of order $h$ in the matrix of the $h m$ coefficients of the $h$ forms on $m>h$ variables. Indeed, $E=1$ if any $D \neq 0, E=0$ if every $D=0$. Since the invariants (32) and (33) vanish for all classes following $B_{r_{1} \ldots \tau_{h}}^{b}$, they are linearly independent. Varying $h$ and the $r$ 's we obtain as many linearly independent invariants (32) and (33) as there are classes $B$. With the class $C_{0}$ of vanishing forms we associate the invariant* 1 .

Since we have exhibited as many linearly independent invariants as there are classes under the group $G_{1}$, we have proved the

Theoren.-For $q>m$, every invariant of $q$ linear forms in the $G F\left[p^{n}\right]$ on $m$ variables is a rational integral function of the invariants of $m$ forms on $m$ variables. A complete set of linearly independent invariants of $q \geqslant m$ forms is given by (30), (32), (33) and unity, each being a polynomial in the determinants of orders $<m$ of the matrix of the coefficients of the forms.

* We may take any invariant, as (35), which does not vanish for $C_{0}$. 
10. For $q<m$, the tirst case of $\S 9$ does not occur. If the $q$ forms are linearly independent, they may be transformed into $x_{1}, \ldots, x_{\eta}$. For the resulting class $B_{1 \ldots q}^{0}$ the characteristic invariant $E_{1 \ldots y}$ is given by (33) for $h=q$. If, for $h<q, h$ of the forms are linearly independent, but every set of $h+1$ dependent, we proceed as in $\$ 9$.

Theorey.-For $q<m$, a complete set of linearly independent invariants of $q$ linear forms on $m$ variables is given by (32) for $h<q$, (33) for $h \leqslant q$, and unity.

11. Instead of introducing the new invariants $E$, we may make use of products of the invariants $A_{i}$ of single forms (\$2). In place of (33), we use

$$
J_{r_{1} \ldots r_{h}}=\operatorname{II} A_{i} \quad\left(i=1, \ldots, q ; \quad i \neq r_{1}, \ldots, i \neq r_{h}\right) .
$$

For the class $C_{0}$ the characteristic invariant is

$$
A_{1} A_{2} \ldots A_{4} \text {. }
$$

For $q \geqslant m$, there are as many invariants (30), (32), (34), (35) as classes under $G_{1}$. Suppose there is a linear (homogeneous) relation between these invariants. For the class $B_{r_{1} \ldots i_{n}}^{0}$, the $D$ 's and $V$ 's vanish, while

$$
A_{r_{1}}=0, \ldots, A_{r_{h}}=0, A_{i}=1 \quad\left(i \neq r_{1}, \ldots, i \neq r_{k}\right) .
$$

Taking $h=m-1$, we see that the only non-vanishing invariant of the set (34), (35) is $J_{r_{1} \ldots r_{m-1}}$, whose coefficient in the relation therefore vanishes. We take in turn the various sets of $m-1$ subscripts $r$. Proceeding similarly with $h=m-2, \ldots, h=1$, we conclude that no invariant (34) occurs in the relations. Taking every $l_{i}=0$, we see that the coefficient of $A_{1} \ldots A_{q}$ vanishes. The relation now involves only the $D^{\prime}$ s and $V^{\prime} \mathrm{s}$; but these were shown in $\$ 9$ to be linearly independent.

For $q<m$, we replace $E_{1 \ldots,}$ of $\$ 10$ by unity, which takes the place of (34) for $h=q$. For the independence proof we employ in succession the classes $B_{r_{1} \ldots r_{h}}^{0}$ for $h=q, q-1, \ldots, 1$ and $C_{0}$. The case $h=q$ shows that the relation lacks the invariant unity.

Theorex. $-A$ complete set of linearly independent invariants of $q$ linear forms on $m$ variables is given by (30), (32), (34), (35) if $q \geqslant m$; and by (32), (34), (35) and unity if $q<m$, viz., by (32) and the $2^{1}$ products $A_{1}^{a_{1}} \ldots A_{1 /}^{a_{q}}($ each $\alpha=0$ or 1$)$.

12. It now follows that the invariant $E$ of $h$ forms on $m$ variables, $m>h$, is expressible in terms of the $A$ 's and $V$ 's. Since $E$ is the 
characteristic invariant for the class containing $x_{1}, \ldots, x_{i}$, formula (14) gives

$$
E_{i j}=\left(1-A_{i}\right)\left(1-A_{j}\right)-V_{i j}^{\mu},
$$

while, by $\$ 2, E_{i}=1-A_{i}$. A comparison of (36) with (9) reveals a fact which is true for any $h$. If, in the expression for $E_{r, ~}$, in terms of the invariants $A$ and $V$ of $h$ forms on $m>h$ variables, we replace $E$ by $D_{r_{1} \ldots r_{h}}^{\mu}$, we obtain a true relation between the invariants of $h$ forms on $h$ variables, where $D$ is the determinant of the latter forms. The converse is true if we employ the non-homogeneous linear relation $D^{\mu}+\ldots=1$ which hold between the invariants of $\$ 11$ for $h$ forms in $h$ variables. Such a relation exists, since the sum of the characteristic invariants is unity $(\$ 1)$ and since the sum of those for the classes $x_{1}, \ldots, x_{h-1}, d x_{h}$ ( $d$ taking all values $\left.\neq 0\right)$ is $D^{\mu}(\$ 4)$. 'This relation not not only gives at once the desired expression for $E$, but is of considerable importance in the general theory. We shall derive it by certain devices for $h=3$ and $h=4$.

13. For three forms in $m \geqslant 3$ variables, we have

\begin{tabular}{|c|c|c|c|c|c|c|c|c|c|c|c|}
\hline & & & $V_{19: 3}$ & $V_{213}$ & $V_{132}$ & $V_{1:}$ & $V_{1:}$ & $V_{: !}$ & $A_{1}$ & $A_{2}$ & $A_{3}$ \\
\hline$x_{1}$ & $x_{2}$ & $b x_{3}$ & 0 & 0 & 0 & 0 & 0 & 0 & 0 & 0 & 0 \\
\hline$x_{1}$ & $x_{2}$ & $c x_{1}+d x_{2}$ & $c$ & $d$ & $-c d^{\mu-1}$ & 0 & $c\left(1-d^{\mu}\right)$ & $d\left(1-c^{*}\right)$ & 0 & 0 & $\left(1-c^{\mu}\right)\left(1-d^{\prime \prime}\right)$ \\
\hline$x_{1}$ & $e x_{1}$ & $x_{2}$ & 0 & 0 & $e$ & $e$ & 0 & 0 & 0 & $1-e^{\omega}$ & 0 \\
\hline 0 & $x_{1}$ & $x_{2}$ & 0 & 0 & 0 & 0 & 0 & 0 & 1 & 0 & 0 \\
\hline$x_{1}$ & $f x_{1}$ & $g x_{1}$ & 0 & 0 & 0 & $f$ & $g$ & $g f^{\mu-1}$ & 0 & $1-f^{\prime \prime}$ & $1-g^{-}$ \\
\hline 0 & $x_{1}$ & $h x_{1}$ & 0 & 0 & 0 & 0 & 0 & $h$ & 1 & 0 & $1-h^{\mu}$ \\
\hline 0 & 0 & $x_{1}$ & 0 & 0 & 0 & 0 & 0 & 0 & 1 & 1 & 0 \\
\hline 0 & 0 & 0 & 0 & 0 & 0 & 0 & 0 & 0 & 1 & 1 & 1 \\
\hline
\end{tabular}

In the first type, $b=1$ if $m>3$; while, if $m=3, b$ may have any value $\neq 0$. The remaining parameters $c, \ldots, h$ are arbitrary in the field. By $\$ 11$ a complete set of linearly independent invariants is given, for $m>3$, by $A_{1}^{a_{1}} A_{2}^{a_{2}} A_{3}^{a_{3}}$ (each $a=0$ or 1 ), the powers of $V_{1: 22}$ and $l_{3: 3}$. the products of the powers of $V_{123}$ and $V_{213}$, and those for $V_{12}$ and $V_{13}$. the exponents being $\leqslant \mu$. For $m=3$, unity is to be deleted and the powers of the determinant $D=D_{123}$ inserted.

Invariant $E_{12}$, defined by (36), equals 1 for the first two types and 0 for the other types. Hence $A_{8} E_{12}$ is the chitracteristic invariant for 
the clags $x_{1}, x_{2}, 0$. To obtain its expression as a linear function of the above invariants, we need $d_{3} V_{12}^{\mu}$. Now

$$
V_{132}^{\mu}-V_{123}^{\mu} V_{213}^{\mu}=V_{13}^{\mu}\left(1-A_{9}-V_{13}^{\mu}\right) \text {, }
$$

since each member vanishes except for the third type and equals $e^{\mu}$ for the latter. Hence

$$
A_{3} E_{12}=A_{9}\left(1-A_{1}\right)\left(1-A_{2}\right)+V_{182}^{\mu}-V_{123}^{\mu} V_{213}^{\mu}-V_{12}^{\mu}+V_{12}^{\mu} V_{13}^{\mu} .
$$

For $m=3, E_{12}-D^{\mu}$ equals 1 for the second type, 0 for the other types. Hence the characteristic invariant for $x_{1}, x_{2}, 0$ is given by

$$
E_{12}-D^{\mu}+\left(1-V_{123}^{\mu}\right)\left(1-V_{213}^{\mu}\right)-1 \text {. }
$$

Inserting the value (36) of $E_{12}$ and equating the result to (38), we obtain the desired non-homogeneous linear relation between our invariants for $m=3$ :

(40) $D^{\mu}=2 V_{123}^{\mu} V_{213}^{\mu}-V_{123}^{\mu}-V_{213}^{\mu}-V_{132}^{\mu}-V_{12}^{\mu} V_{13}^{\mu}+\left(1-A_{1}\right)\left(1-A_{2}\right)\left(1-A_{3}\right)$.

The right member is thus the expression for the invariant $E_{129}$ of three forms in $m$ variables for $m>3$.

We note in passing that it is not difficult to exhibit the characteristic invariant $I_{c, d}$ of the general class $A_{c, d}$ of the second type. As in $\S 4$, we employ

$$
\kappa_{c}=-\sum_{k=1}^{\mu} c^{-k} V_{128}^{k}, \quad \lambda_{d}=-\sum_{k=1}^{\mu} d^{-k} V_{218}^{k}
$$

If $c \neq 0, \kappa_{c}=1$ for $A_{c, d}, 0$ for $A_{\gamma, \delta}$, for every $d, \gamma, \delta, \gamma \neq c$. If $d \neq 0, \lambda_{d}=1$ for $A_{c, d}, 0$ for $A_{\gamma, \delta}$, for every $c, \gamma, \delta, \delta \neq d$. Hence

$$
\begin{aligned}
& I_{c, d}=\kappa_{c} \lambda_{d} \quad(c d \neq 0), \quad I_{c, 0}=\kappa_{c}\left(1-V_{213}^{\mu}\right) \quad(c \neq 0), \\
& I_{0, d}=\lambda_{d}\left(1-V_{123}^{\mu}\right) \quad(d \neq 0), I_{0,0} \text { in (38) or (39). }
\end{aligned}
$$

As a check, the sum of these $p^{2 n}$ characteristic invariants was verified to be $E_{12}-D^{\mu}$ by means of $\Sigma c^{-k} d^{-t}=\left(\Sigma c^{-k}\right)\left(\Sigma d^{-t}\right)=1$ if $\kappa=t=\mu$, otherwise $=0$, where the sums range over all elements $c \neq 0, d \neq 0$.

14. For 4 forms in 4 variables, we obtain two determinations of the characteristic invariant $I$ for the class $x_{1}, x_{2}, x_{3}, 0$. As in (39),

$$
I=E_{123}-D^{\mu}+\left(1-V_{1234}^{\mu}\right)\left(1-V_{2134}^{\mu}\right)\left(1-V_{3124}^{\mu}\right)-1,
$$

where $E_{129}$ is the right member of (40). Again, $I=A_{4} E_{123}$. As in (37),

$$
V_{1243}^{\mu}-V_{1234}^{\mu} V_{3124}^{\mu}=V_{123}^{\mu}\left(1-A_{4}-V_{124}^{\mu}-V_{214}^{\mu}+V_{124}^{\mu} V_{214}^{\mu}\right) \text {. }
$$

Hence $A_{4} V_{123}^{\mu}$ is expressed linearly in terms of our system of invariants (§11). Interchanging subscripts 1,2 or 2,3 , we get $A_{4} V_{2: 3}^{\mu}, A_{4} V_{182}^{\mu}$. 
Multiplying (44) by $V_{213}^{\mu}$ and applying

$$
V_{213} V_{3124}=0, \quad V_{213}^{\mu} V_{1243}^{\mu}=V_{1243}^{\mu} V_{3143}^{\mu}-V_{1234}^{\mu} V_{2134}^{\mu} V_{3124}^{\mu},
$$

we obtain $A_{4} V_{123}^{\mu} V_{218}^{\mu}$ linearly in terms of the system. It remains to find $A_{4} V_{12}^{\mu} V_{13}^{\mu}$. Interchanging 3 and 4 in (37), we have

$$
A_{4} V_{12}^{\mu}=V_{12}^{\mu}-V_{12}^{\mu} V_{14}^{\mu}+W, \quad W \equiv V_{124}^{\mu} V_{214}^{\mu}-V_{142}^{\mu} \text {. }
$$

To find $V_{13}^{\mu} W$, we note that $V_{13}^{\mu} W+V_{142}^{\mu} V_{143}^{\mu}$ vanishes for every class except that containing $x_{1}, x_{2}, a x_{1}+b x_{2}, c x_{1}+d x_{2}$; and for it has the value $c^{\mu} d^{\mu} \Delta^{\mu}$, where $\Delta=b c-a d$. But

$$
P=V_{213} V_{124}-V_{123} V_{214}
$$

vanishes except for the same class, and for it has the value $\Delta$. Thus

$$
V_{13}^{\mu} W=P^{\mu} V_{124}^{\mu} V_{214}^{\mu}-V_{142}^{\mu} V_{143}^{\mu} \text {. }
$$

To $P^{\mu}$ we apply (5). By additions we obtain $A_{4} E_{123}$ expressed linearly in the system. Comparing the result with (43), we obtain

$$
\begin{aligned}
D^{\mu}=(1 & \left.-A_{1}\right)\left(1-A_{2}\right)\left(1-A_{3}\right)\left(1-A_{4}\right)-3 V_{1234}^{\mu} V_{2134}^{\mu} V_{3124}^{\mu} \\
& +2\left(V_{1234}^{\mu} V_{2134}^{\mu}+V_{1234}^{\mu} V_{3124}^{\mu}+V_{2134}^{\mu} V_{3144}^{\mu}\right)+2 V_{1243}^{\mu} V_{2143}^{\mu}-V_{1: 43}^{\mu} \\
& -V_{2143}^{\mu}-V_{1234}^{\mu}-V_{2134}^{\mu}-V_{3124}^{\mu}-V_{1342}^{\mu}-2 V_{123}^{\mu} V_{: 13}^{\mu} V_{124}^{\mu} V_{: 114}^{\mu} \\
& -V_{12}^{\mu} V_{13}^{\mu} V_{14}^{\mu}-\left(V_{123}^{\mu} V_{124}^{\mu}+V_{123}^{\mu} V_{214}^{\mu}+V_{21:}^{\mu} V_{124}^{\mu}+V_{213}^{\mu} V_{214}^{\mu}\right) \\
& -\left(V_{132}^{\mu} V_{134}^{\mu}+V_{132}^{\mu} V_{314}^{\mu}\right)-V_{142}^{\mu} V_{143}^{\mu}+V_{132}^{\mu} V_{134}^{\mu} V_{314}^{\mu} \\
& +2 V_{123}^{\mu} V_{213}^{\mu}\left(V_{124}^{\mu}+V_{214}^{\mu}\right)+2 V_{124}^{\mu} V_{214}^{\mu}\left(V_{123}^{\mu}+V_{213}^{\mu}\right) \\
& +\sum_{r=1}^{\mu}\left(V_{123} V_{214}\right)^{r}\left(V_{213} V_{124}\right)^{\mu-r}
\end{aligned}
$$

the terms in any parenthesis being related under the classitication in $\$ \mathbf{9}$. The second member of (45) defines $E_{1231}$ for $m>4$ variables.

15. In addition to the general method in $\S 8$ of constructing the invariants $V$, a second method was employed in $\$ 3$ for the case of two forms. As the latter method, apart from details, is that commonly used in the algebruic theory, it will prove interesting to give the results obtained similarly for three forms. In (2) we replace $a_{i v}$ by $a_{i v}-\kappa a_{j v}-\lambda a_{k v}$ and, in view of (3), express the result in the form

$$
\mathrm{II}=A_{i}\left[1+\left(A_{j}-1\right) \kappa^{\mu}\right]\left[1+\left(A_{k}-1\right) \lambda^{\mu}\right]-\sum_{t=1}^{\mu} V_{i j}^{t} \kappa^{t}-\sum_{t=1}^{\mu} V_{i k}^{t} \lambda^{t}+\sum_{i, s=1}^{\mu} T_{r s} \kappa^{r} \lambda^{*},
$$

which, for $\lambda=0$, reduces to (4). We obtain the value of $T_{r s}$ for each canonical set of the three forms ( $\$ 13)$. Most of the cases may be treated 
444 Modular invariants of a GENERAL SYSTEM OF LiNEaR FORMS.

by inspection. But for the second type

$$
\begin{aligned}
\Pi & =\left[1-(1-c \lambda)^{\mu}\right]\left[1-(-\kappa-d \lambda)^{\mu}\right] \\
& \equiv\left[\sum_{t=1}^{\mu} c^{t} \lambda^{t}\right]\left[d^{\mu} \lambda^{\mu}-1+\sum_{r=1}^{\mu}(-1)^{r} \kappa^{r}(d \lambda)^{\mu-r}\right] \quad(\bmod p),
\end{aligned}
$$

by (5). For the fourth type

$$
\begin{aligned}
\Pi & =1-(1-f \kappa-g \lambda)^{\mu} \equiv-\sum_{l=1}^{\mu}(f \kappa+g \lambda)^{l} \\
& =-\Sigma\left(\begin{array}{c}
r+s \\
r
\end{array}\right) f^{r} g^{s} \kappa^{r} \lambda^{s} \quad(r, s=0,1, \ldots, \mu ; r+s=1, \ldots, \mu) .
\end{aligned}
$$

We find that $T_{r s}=0$ for types $1,4,7,8 ;(-1)^{r} c^{r+s} d^{\mu-r}$ for 2 ; $e^{r}(s \doteq \mu), 0(s<\mu)$ for type $3 ;-\left(\begin{array}{c}r+s \\ r\end{array}\right) f^{r} g^{s}(r+s \leqslant \mu), 0(r+s>\mu)$ for 5 ; $(-1)^{s-1} h^{s}(r+s=\mu$ or $r=s=\mu)$, otherwise 0 for type 6 . Hence $T_{\mu 1}=V_{i j k}, T_{1 \mu}=V_{i k j}$. Permuting the forms $l_{i}$ and $l_{j}$, we see that the new function $T_{\mu 1}$ is $V_{j i k}$. Conversely, each $T_{r s}$ may easily be expressed in terms of the $V$ 's and $A$ 's. As a complete set of linearly independent invariants we may take

$$
\begin{array}{r}
T_{r s}, V_{i j}^{r}, V_{i k}^{r}, V_{j k}^{r}, V_{i j}^{r} V_{i k}^{s}, A_{i} V_{j k}^{r}, A_{j} V_{i k}^{r}, A_{k} V_{i j}^{r}, A_{i}^{a_{i}} A_{j}^{a_{j}} A_{k}^{a_{k}} \\
\left(r, s=1, \ldots, \mu ; \alpha^{\prime} \mathrm{s}=0,1\right),
\end{array}
$$

if $m>3$; the same with unity deleted and $D^{r}$ inserted, if $n=3$. The only non-homogeneous linear relation between the invariants for $m=3$ is

$$
D^{\mu}+T_{\mu \mu}+V_{j k}^{\mu}=\left(1-A_{\imath}\right)\left(1-A_{j}\right)\left(1-A_{k}\right)
$$

